\title{
Glass Model, Hubbard Model and High-Temperature Superconductivity
}

\author{
Ingo Morgenstern, Werner Fettes, Thomas Husslein, \\ Fakultät für Physik, Universtät Regensburg, 93053 Regensburg, Germany \\ Dennis M. Newns, and Pratap C. Pattnaik \\ IBM T.J. Watson Research Center, P.O. Box 218, Yorktown Heights, NY 10598, USA
}

(September 2, 2018)

In this paper we revisit the glass model describing the macroscopic behavior of the High-Temperature superconductors. We link the glass model at the microscopic level to the striped phase phenomenon, recently discussed widely. The size of the striped phase domains is consistent with earlier predictions of the glass model when it was introduced for High-Temperature Superconductivity in 1987. In an additional step we use the Hubbard model to describe the microscopic mechanism for $d$-wave pairing within these finite size stripes.

We discuss the implications for superconducting correlations of Hubbard model, which are much higher for stripes than for squares, for finite size scaling, and for the new view of the glass model picture.

Keywords: High- $T_{c}$, Superconductivity, Glass Model, Hubbard Model, Finite Size Scaling, Pseudo-Gap

\section{INTRODUCTION AND GLASS MODEL}

More then 10 years have passed since the introduction of the glass model in High-Temperature Superconductivity [1 [8]. The model was originally used for the description of granular superconductors [9]. But it became immediately clear that the glass behavior of the then new High- $T_{c}$ materials was not caused by the granular structure of the ceramic compounds; rather the magnetic fields used in the experiments suggested that the microscopic origin of glassy behavior occurred at a length scale smaller than that of the grains [1].

In this paper we revisit the glass model. Inspired by the recent study by Tsuei and Doderer 10 of the charge confinement effect in cuprate superconductors, we link the intragrain inhomogeneities demanded in the glass theory to the microscopic concept of striped phase [11,12, and in particular to that of stripe domains [13, 10 which have recently become of wider interest to the High$T_{c}$ community. The stripe domains are identified with the areas of constant phase in the glass theory. Microscopically, Tsuei and Doderer used their charge confinement model [10] to explain various generic features of the normal-state pseodogap, including the magnitude of the gap. Such stripe domains may be described from first principles by a model such as the Hubbard Model, enabling an ab initio numerical calculation of intra-domain pairing properties such as the effective pairing temperature and pseudogap.
In [1] a behavior reminiscent of spin glasses was detected, the quasi-de-Almeida-Thouless-line (quasi ATline):

$$
H^{2 / 3} \approx T_{c}(H)-T_{c}(0)
$$

where $H$ is the magnetic field and $T_{c}(H)$ the corresponding critical temperature or to be more precise the temperature below which metastable behavior occurs as seen in [7]. The existence of the AT-line was first seen as an evidence for glass behavior, but it turned out, that a totally different physical mechanism was the cause for the $\mathrm{H}^{2 / 3}$ behavior [1]. Numerical simulations of the High- $T_{c}$ glass model [1] lead to this conclusion.

The glass model can be described by the Hamiltonian |1

$$
\mathcal{H}=-J \sum_{<i, j>} \cos \left(\phi_{i}-\phi_{j}-A_{i j}\right)
$$

where

$$
A_{i j}=\int_{i}^{j} \vec{A} d l
$$

$\vec{A}$ is the vector potential leading to 1

$$
A_{i j}=\frac{2 \pi}{\phi_{0}} H \frac{x_{i}+x_{j}}{2}\left(y_{j}-y_{i}\right) \text {. }
$$

In equation (2) to (4) $\phi_{0}$ is the elementary flux quantum, $H$ the magnetic field. $\phi_{i}$ is the phase of the superconducting wave function in an area or domain denoted by i; $x_{i}$ and $y_{i}$ are the cartesian coordinates. Furthermore $J$ is the coupling energy between two clusters according to [9] taken identical in the simulations in [1], $\int_{i}^{j}$ the line integral from cluster $i$ to $j,\langle i, j\rangle$ the sum over the nearest neighbors, and finally $A_{i j}$ are the phase factors between cluster $i$ and $j$.

As a result of the simulations in [1] we consider figure 1] which is reproduced from [1] and follow-up papers. Because of the results of the simulations we had to conclude, that the origin of the glass behavior lies inside the grains, therefore also inside single crystals.

The $H^{2 / 3}$ behavior was only reproduced in two dimensions, therefore the domains or areas of constant phase $\phi_{i}$ had to be located inside the $\mathrm{CuO}$-planes, connected by weak links $J$ (figure 1 ). The size of the domains was 
also known. Here we present a typical calculation of estimating the domain size with data available at the time of the publication of 1,14 .

As the magnetic field of reference we consider the upper critical field $H_{c}^{u}$. In figure 2 we see $H_{c}^{u}$ in the phase diagram of equation (11), that $H_{c}^{u} \approx 0.5$. We should denote the $2 \pi / \phi_{0}$ was taken as unity as is normal in numerical simulations.

For an estimation of the typical distance $a$ between two clusters, we consider a plaquette in figure 3 .

In the simulation $a=1$. The idea is that the phase factors are equal, $A_{i j, s i m}=A_{i j, e x p}$, for corresponding fields $H$. $\left(A_{i j, s i m}\right.$ denoting $A_{i j}$ used in simulation and $A_{i j, \exp }$ correspondingly in experiment). Therefore for $H_{c}^{u}$ we have $A_{i j, s i m}=0.5$. Considering the experimental situation we refer to figure 1 in 14. There the magnetization or magnetic moment $\mu(H)$ is plotted versus $H$ for various temperatures. Considering the lowest temperature $\mu(H)$ tends to zero at $1.5 T$. Therefore $H_{c}^{u} \approx 1.5 T$ (Tesla) in experiment with $\phi_{0}=2.06 \cdot 10^{-15} \mathrm{~m}^{2} \mathrm{~T}$ we find that $a^{2} \approx 1 \cdot 10^{4} \AA^{2}$ and therefore $a \approx 100 \AA$.

We wish to emphasize here, that the length scale of $100 \AA$ deduced from the glass picture is consistent with the length of the stripe domains seen in STM data [13], and roughly with that of the stripe width determined from neutron 11 and ultraviolet photoemission 12 data. We propose revision of the concept of clean, single crystal high temperature superconductor material to include the concept of striped phase-type inhomogeneity with associated 'glassy' behavior. It should be noted, that the aging or memory effect in spin glasses was also detected [8].

At the end of this section we would like to counter a certain source of criticism concerning the glass model. Ten years ago the community only considered the case of s-wave superconductivity. In the model $J=1$ was chosen. The glass behavior as described in [1] is totally dominated by frustration, which is caused by the $A_{i j}$ 's in equation (2). In s-wave superconductivity $J$ would not change sign and therefore not lead to additional frustration. This could be different in $\mathrm{d}$-wave as pointed out by Sigrist and Rice 15.

But the situation in the simulation of [1] was, that only weak "correlated" disorder was chosen in the framework of the square lattice. This means the coordinates $x_{i}$ and $y_{i}$ in equation (4) were obtained by allowing a random displacement of the sites within a circle with radius $r \approx 0.4$ of the lattice constant around the original site of the square lattice. Therefore $J$ does not change sign even in the case of d-wave superconductivity. It should be pointed out that only this type of weak "correlated" disorder [1] in two dimensions reproduced the $H^{2 / 3}$ behavior of the quasi AT-line [14]. At that time this result also lead to the prediction of a roughly two-dimensional plane-like anisotropy in the new superconductors [1]
Summarizing the glass model picture of [1] is still applicable to the correlated striped domains in the High- $T_{c}$ materials governing the d-wave symmetry and predicting the domain sizes correctly.

\section{MICROSCOPIC MECHANISM AND HUBBARD MODEL}

As pointed out the microscopic mechanism has to be considered inside the domains. While glass papers did not deal with the mechanism, it was clear at that time, that, if the glass picture applies, the observed $T_{c}^{e x p}$ is $T_{c}^{\text {glass }}$ of the glass model. The microscopic mechanism could easily have a much higher critical temperature $T_{c}^{m}$.

Inside the domains we propose an electronic mechanism as suggested by the Hubbard model (HM) or to be more precise the $t^{\prime}{ }^{-}-\mathrm{HM} 16,17$

$$
\mathcal{H}=\mathcal{H}_{\text {kin }}+\mathcal{H}_{\text {pot }}
$$

with the kinetic

$$
\mathcal{H}_{k i n}=-\sum_{i, j, \sigma} t_{i, j}\left(c_{i, \sigma}^{\dagger} c_{j, \sigma}+c_{j \sigma}^{\dagger} c_{i \sigma}\right)
$$

and the potential part

$$
\mathcal{H}_{\text {pot }}=U \sum_{i}\left(n_{i, \uparrow}-\frac{1}{2}\right)\left(n_{i, \downarrow}-\frac{1}{2}\right)
$$

of the Hamiltonian. We denote the creation operator for an electron with spin $\sigma$ at site $i$ with $c_{i, \sigma}^{\dagger}$, the corresponding annihilation operator with $c_{i, \sigma}$, and the number operator at site $i$ with $n_{i, \sigma} \equiv c_{i, \sigma}^{\dagger} c_{i, \sigma} \cdot t_{i, j}$ is the hopping parameter only nonzero between nearest neighbors $i, j$ $\left(t_{i, j}=t\right)$ and next nearest sites $i, j\left(t_{i, j}=t^{\prime}\right)$, and finally $U$ is the interaction being repulsive in the High- $T_{c}$ case.

The sites of the HM can be associated with the copper in the $\mathrm{CuO}$-layers of the High- $T_{c}$ crystals [18].

We published evidence for superconducting behavior with d-wave symmetry of the repulsive HM as early as 1994 [16]. The d-wave symmetry in the superconducting state was subsequently established for the High- $T_{c}$ materials [19].

According to 20,21] we use the (vertex) correlation function (instead of the largest eigenvalue) to provide evidence for superconductivity following the standard concept of off diagonal long range order 22. The vertex correlation function (vertex $\mathrm{CF}$ ) is defined for $\mathrm{d}_{x^{2}-y^{2-}}$ waves as

$$
C_{d}^{V}(r)=C_{d}(r)-\frac{1}{L} \sum_{i, \delta, \delta^{\prime}} g_{\delta} g_{\delta^{\prime}} C_{\uparrow}(i, i+r) C_{\downarrow}\left(i+\delta, i+r+\delta^{\prime}\right)
$$


with the single particle correlation function $C_{\sigma} \equiv$ $\left\langle c_{i, \sigma}^{\dagger} c_{i+r, \sigma}\right\rangle$ for spin $\sigma$ to extract the pairing effects in the two particle $\mathrm{CF}$

$$
C_{d}(r)=\frac{1}{L} \sum_{i, \delta, \delta^{\prime}} g_{\delta} g_{\delta^{\prime}}\left\langle c_{i \uparrow}^{\dagger} c_{i+\delta \downarrow}^{\dagger} c_{i+r+\delta^{\prime} \downarrow} c_{i+r \uparrow}\right\rangle
$$

with the phase factors $g_{\delta}, g_{\delta}^{\prime}= \pm 1$ to model the $\mathrm{d}_{x^{2}-y^{2}-}$ wave symmetry, the number of lattice points $L$ and the sum $\delta\left(\delta^{\prime}\right)$ over all nearest neighbors.

In figure 1 we reproduce the evidence for d-wave pairing in the repulsive HM using the projector quantum Monte Carlo (PQMC) technique. These simulations 23,24 performed for the relatively weak-coupling value of $U=2$, were found, with adequate numerical precautions, to be quite stable 25 27. For higher $U$-values, e.g. $U=4, \mathrm{PQMC}$ is found to encounter difficulties for larger than $8 \times 8$ cluster sizes, and in fact evidence for finite plateaus was not reproduced, e.g. [28 30], for large $U$. However in the simulation technique of Zhang, Carlson and Gubernatis 31] the existence of the plateau phenomenon in the vertex CF (figure was confirmed. Recent Variational Monte Carlo results 32 also confirm the existence of d-wave pairing in Hubbard clusters.

Given the observation of plateaus supporting the existence of $\mathrm{d}$-wave pairing in finite clusters, what are the implications for bulk superconductivity? Up to now it was thought necessary to scale the finite cluster calculations to infinite size in order to answer this question, which unfortunately presents difficulties as corrections to scaling are tremendously large compared to classical systems 33,26, 34. However in view of the new stripe phase picture of the crystalline High- $T_{c}$ material, the simulated cluster can be reinterpreted as a single finite length stripe as seen in STM data [13], with typical dimensions of roughly $12 \times 4$ unit cells, i.e. even smaller than our largest system sizes $(16 \times 16$ in 34$)$. Scaling to an infinite system is inappropriate as the PQMC cluster calculation is now descriptive of the actual quantum systems present in the crystal (although they are treated as totally isolated).

We consequently performed additional simulations for $12 \times 4$ systems and now report the remarkable result that the plateau $(4 \times 12)$ is about a factor of five higher than the plateau $(12 \times 12)$, figure 5 . We averaged only the vertex $\mathrm{CF} C_{d}^{V}(r)$ in the large range regime of $r$, i. e. for the distances $|r|>\left|r_{c}\right|$ :

$$
\bar{C}_{d}^{V, P} \equiv \frac{1}{L_{c}} \sum_{r,|r|>\left|r_{c}\right|} C_{d}^{v}(r)
$$

with the number $L_{c}$ of points with $|r|>\left|r_{c}\right|$. The qualitative behavior of our results is not influenced by the choice of $r_{c}$ as long as we suppress the short range correlations (i. e. $r_{c} \geq 1.9$ ).

Due to finite size effects (see [35,34 or in more detail [26]) the shape of the curves in respect to the filling in figure 5 is influenced by $\langle n\rangle$ and $L$.
Considering the estimates of the microscopic critical temperature $T_{c}^{m}$ from grand canonical Monte Carlo (GQMC) 36] simulations for the squared finite Hubbard model in 33] we can calculate $T_{c}^{m}$ from the $\chi^{V, P} \equiv$ $L \bar{C}_{d}^{V, P}$ versus $\beta$ plot. ( $\beta$ is the inverse temperature.) In figure 6 we reproduce the results of the GQMC runs in 33. We take as estimate of $T_{c}^{m}$ the inflection point of the $\chi_{d}^{V, P}$ versus $\beta$ curve. From figure 6 we deduce $\beta$ to be roughly $\beta \approx 10$. Taking the hopping parameter $t=0.1 \mathrm{eV}$ this corresponds to the temperature $T_{c} \approx 100 K$.

In figure 6 all curves for different system sizes intersect at one temperature. Even so the magnitude of the superconducting signal at low temperatures is increased for striped geometries, we expect from figure 6 , that the value of $T_{c}^{m}$ should be approximately the same. Here " $T_{c}$ " denotes the temperature where the correlation length equals the size of the systems.

The first occurrence of preformed pairs in the finite Hubbard clusters is at an even higher temperature $T^{*}$. $T^{*}$ is at the onset of the susceptibility, i.e. the temperature with the first occurrence of a nonzero susceptibility, in figure 6. A rough estimate is $\beta \approx 5$. With $t=0.1 \mathrm{eV}$ this corresponds to $T^{*} \approx 200 \mathrm{~K}$, which is in agreement with experimental results 37 . It should be noted, that the range for $t$ is between $0.1 \mathrm{eV}$ to $1 \mathrm{eV}$ as deduced from the experiments 4244 , thus this onset could be even at higher temperatures [45].

Recently Tsuei and Doderer [10], have pointed out a possible interpretation of the pseudogap as a function of doping in cuprates as the finite size gap in the quasiparticle spectrum of the stripe phase domains. Consistent with these ideas, we also identify the onset temperature $T^{*}$ with the pseudogap energy in the cuprate material. Numerical values for the energy gap between the ground state and the first excited state in finite size cluster systems were obtained already in 1995 [46. Figure 7 reproduces a typical case for an $\sqrt{8} \times \sqrt{8}$ system as seen in Lanczos diagonalization (the finite size gap is large for the relatively small clusters for which Lanczos diagonalization is tractable). The experimentally seen superconducting gap is different from the pseudogap (finite size gap). It is the gap seen in the macroscopic glass model when the system as a whole becomes superconducting and the finite gaps are correlated. Therefore it is clear that for the superconducting (glass model) gap a clear d-wave symmetry is seen [19] while in the case of the pseudogap the effect is smeared out 38,40 as the experiment "averages" over different (finite size) clusters and slight correlated disorder. 


\section{SUMMARY AND CONCLUSIONS}

The new view of High- $T_{c}$ superconductivity emerging from Ref. 10 and the present paper is based macroscopically on the interaction of nanoscopic, internally-paired, domains (glass model) and microscopically on the enhancement of pairing found to occur within metallic domains constituting the striped phase. The glass model for example enables us to identify the superconducting $T_{c}$ with the $T_{c}^{\text {glass }}$ of the glass model.

The microscopic model of a domain can be exploited, based on a Hamiltonian such as the Hubbard model, to estimate the relatively high temperature $T^{*}$, the effective $\mathrm{d}$-wave intra-domain pairing temperature, and to determine the pseudogap which is identified [10] with the finite size quasiparticle gap within the domain. In earlier simulations we already found the first signs of pairing in the Hubbard model at remarkably high temperatures 33. We find in the present work a very large enhancement of the intra-domain pairing when a non-square cluster morphology, $12 \times 4$, appropriate for the striped phase, is considered.

A further consequence of the glass model is that all changes in $T_{c}^{e x p}$ are related to the interdomain coupling energy $J$ of equation (2) and not to the microscopic mechanism described by the Hubbard model. This means, e.g. for the isotope effect, that when masses are altered by changing $\mathrm{O}^{16}$ to $\mathrm{O}^{18}$, leading to the isotope exponent $\alpha=\Delta T_{c} / \Delta M$, the mass effect is entering through an isotope shift in $J$ or other glass model parameters. $T^{*}$ is probably not affected by the isotope effect 47]. This would be another evidence for the combined glass model - Hubbard model picture.

In general we note that all experiments aiming at changing $T_{c}^{m}$ and therefore the microscopic mechanism have to be reinterpreted or redone in terms of $J$ of the glass model. Future research could usefully concentrate on these weak links as seems to be occurring [48. Only the glass model $J$ governs the $T_{c}^{\exp }$ (in fact $T_{c} \approx J$ ), and additionally the critical currents of the materials. Progress in increasing $T_{c}$ or the critical current so far has been only accidental as the underlying glass behavior was not recognized.

In the scientific discussion about ten years ago it was concluded [7] that the glass model would be only applicable to so called "bad" or "glassy" samples, i.e. samples with weak links described by Hamiltonian of equation (2) [7]. At this time future research had to concentrate on "good" samples without weak links. Here we argue again, that there are no "good" samples, as the $T_{c}^{\text {exp's }}$ for all samples, single crystals, thin films, and ceramics, are for a definite composition about the same. It follows consequently that the $T_{c}^{\exp }$ is the $T_{c}^{\text {glass }}$ of the glass model.

Furthermore it is clear as we deal with finite size do- mains (or stripes) that mean field theory and therefore BCS does not apply.

Future research could usefully emphasize the origin and manipulation of the weak links (i.e. the $J$ in the glass model) between the domains (or stripes).

\section{ACKNOWLEDGMENT}

The authors would like to express their thanks to C.C. Tsuei and T. Doderer for making available a preprint of their work [10]. We would like to acknowledge very helpful discussions with T. Schneider, H. Keller, D. Brinkman, Ch. Rossel, J.G. Bednorz, J.P. Loquet, H. De Raedt and U. Krey. Especially we would like to thank K.A. Müller for inspiring discussions and ideas. In addition, W. F. is grateful for the financial support of the DFG (Deutsche Forschungsgemeinschaft). A part of the numerical simulations were performed on the SP2 parallel computer of the Leibnitz Rechenzentrum Munich, which grants us a generous amount of CPU time. Finally we acknowledge the financial support of the UniOpt GmbH, Regensburg.

[1] I. Morgenstern, K.A. Müller, and J.G. Bednorz. Z. Phys. B69, 33 (1987).

[2] J.W. Schneider, Hp. Baumeler, H. Keller, W. Obermatt, B.D. Patterson, K.A. Müller, J.G. Bednorz, K.W. Blazey, I. Morgenstern, and I.M. Savic. Phys. Letters, A124, 107 (1987).

[3] I. Morgenstern. Physica, C153-155, 59 (1988).

[4] B. Pümpin, H. Keller, W. Kündig, W. Odermatt, B.D Patterson, J.W. Schneider, H. Simmler, S. Connell, K.A. Müller, J.G. Bednorz, K.W. Blazey, I. Morgenstern, C. Rossel, and I.M. Savic. Z. Phys., B72, 175 (1988).

[5] I. Morgenstern, K.A. Müller, and J.G. Bednorz. Physica, B152, 85 (1988).

[6] H. Keller, P. Pümpin, W. Kündig, W. Obermatt, B.D. Patterson, J.W. Schneider, K.A. Müller, J.G. Bednorz, K.W. Blazey, I. Morgenstern, C. Rossel, and I.M. Savic. Physica, C153-155, 71 (1988).

[7] I. Morgenstern. IBM Journal of Research and Development, 33, 307 (1989).

[8] C. Rossel, Y. Maeno, and I. Morgenstern. Phys. Rev. Letters, 62, 681 (1989).

[9] C. Ebner and D. Stroud. Phys. Rev., B31, 165 (1985).

[10] C.C. Tsuei and T. Doderer. 'Charge confinement effect in cuprate superconductors - an explanation for the normalstate resistivity and pseudogap', preprint, 1998.

[11] J.M. Tranquada, J.D. Axe, N Ichikawa, A.R. Moodenbaugh, Y. Nakamura, and S. Uchida. Phys. Rev. Letters, 78, 338 (1997).

[12] N.L. Saini, A. Lanzara, H. Oyanagi, H. Yamaguchi, 
K. Oka, T. Ito, and A. Bianconi. Phys. Rev., B55, 12759 (1997).

[13] C.J. Chen and C.C. Tsuei. Solid State Communication, 71, 33 (1989).

[14] K.A. Müller, M. Takashige, and J.G. Bednorz. Phys. Rev. Letter, 58, 1143 (1987).

[15] M. Sigrist and T.M. Rice. J. Phys. Soc. Jpn., 61, 4283 (1992).

[16] I. Morgenstern, W. Fettes, T. Husslein, C. Baur, H.G. Matuttis, and J.M. Singer. Proc. PC94 Conference, Lugano, 1994.

[17] T. Husslein, I. Morgenstern, D.M. Newns, P.C. Pattnaik, and J.M. Singer. Phys. Rev, B54, 16179 (1996), and references therein.

[18] P.W. Anderson. Science, 235, 1196 (1987).

[19] C.C. Tsuei, J.K. Kirley, C.C. Chi, L.S. Yu-Jahnes, A. Gupta, T. Shaw, J.Z. Sun, and M.B. Ketchen. Phys. Rev. Lett., 73, 593 (1994).

[20] R.T. Scalettar, E.Y. Loh, J.E. Gubernatis, A. Moreo, S.R. White, D.J. Scalapino, R.L. Sugar, and E. Dagotto. Phys. Rev. Lett., 62, 1407 (1989).

[21] M. Frick, I. Morgenstern, and W. von der Linden. Z. Phys., B82, 339 (1991).

[22] C.N. Yang. Rev. Mod. Phys., 34, 694 (1962).

[23] G. Sugiyama and S.E. Koonin. Ann. Phys., 168, 1 (1986).

[24] S. Sorella, A. Parola, M. Parrinello, and E. Tosatti. Int. J. Mod. Phys., B3, 1875 (1989).

[25] T. Husslein, W. Fettes, and I. Morgenstern. Int. J. Mod. Phys., C8, 397 (1997).

[26] W. Fettes. Ph.D. Thesis, Universität Regensburg, 1998.

[27] W. Fettes and I. Morgenstern. accepted by European Physical Journal B, 1998.

[28] J.E. Hirsch. Phys. Rev., B38, 12023 (1988).

[29] S.R. White, D.J. Scalapino, R.L. Sugar, and N.E. Bickers. Phys. Rev. Lett., 63, 1523 (1989).

[30] M. Imada. J. Phys. Soc. Jpn., 60, 2740 (1991).

[31] S. Zhang, J. Carlson, and J.E. Gubernatis. Phys. Rev. Lett., 78, 4486 (1997).

[32] K. Yamaji, T. Yanagisawa, T. Nakanishi, and S. Koike, preprint, 1998

[33] T. Husslein. Ph.D. Thesis, Universität Regensburg, 1996.

[34] W. Fettes and I. Morgenstern. Int. J. Mod. Phys., C9, 943 (1998).

[35] W. Fettes, I. Morgenstern, and T. Husslein. Int. J. Mod. Phys., C8, 1037 (1997).

[36] R. Blankenbecler, D.J. Scalapino, and R.L. Sugar. Phys. Rev., D24, 2278 (1981).

[37] H. Ding, T. Yokoya, J.C. Campuzano, T. Takahashi, M. Randeria, M.R. Norman, T. Mochiku, K. Kadowaki, and J. Giapintzakis. Nature, 382, 51 (1996).

[38] A.G. Loeser, Z.-X. Shen, D.S. Dessau, D.S. Marshall, C.H. Park, P. Fournier, and A. Kapitulnik. Science, 273, 325 (1996).

[39] A.G. Loeser, D.S. Dessau, and Z.-X. Shen. Physica, C263, 208 (1996).

[40] J.M. Harris, Z.-X. Shen, P.J. White, D.S. Marshall and M.C. Schabel, J.N. Eckstein, and I. Bozovic. Phys. Rev., B54, R15665 (1996).

[41] H. Keller, D. Brinkman, and T. Schneider. private communication, 1997.
[42] M.S. Hybertsen, M. Schlüter, and N.E. Christensen. Phys. Rev., B39, 9028 (1989).

[43] S.B. Bacci, E.R. Gagliano, R.M. Martin, and J.F. Annett. Phys. Rev., B44, 7504 (1991).

[44] J. Song and J.F. Annett. Phys. Rev., B51, 3840 (1995).

[45] D.M. Newns. private communication, 1998.

[46] W. Fettes, I. Morgenstern, T. Husslein, H.-G. Matuttis, J.M. Singer, and C. Baur. J. Phys. I France, 5, 455 (1995).

[47] H. Keller. private communication, 1997.

[48] O. Zachar, S.A. Kivelson, and V.J. Emery. Phys. Rev., B57, 1422 (1998).

(1)

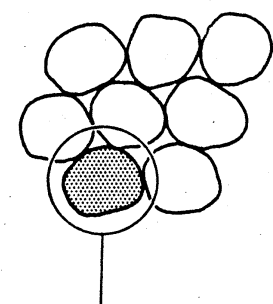

3D array
of grains

(2)

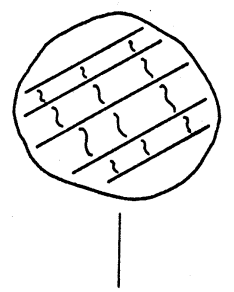

2D planes:

inside grain

(3)

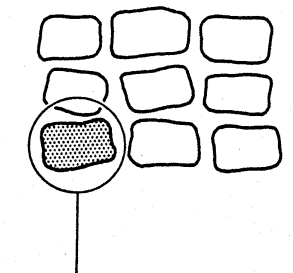

Plane: domains (twins)

(4)

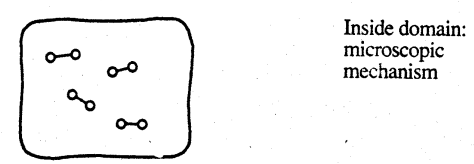

FIG. 1. Schematic illustration of the physical situation in a single crystal of a High- $T_{c}$ ceramic. (analogous to figure 1 in [7]) 


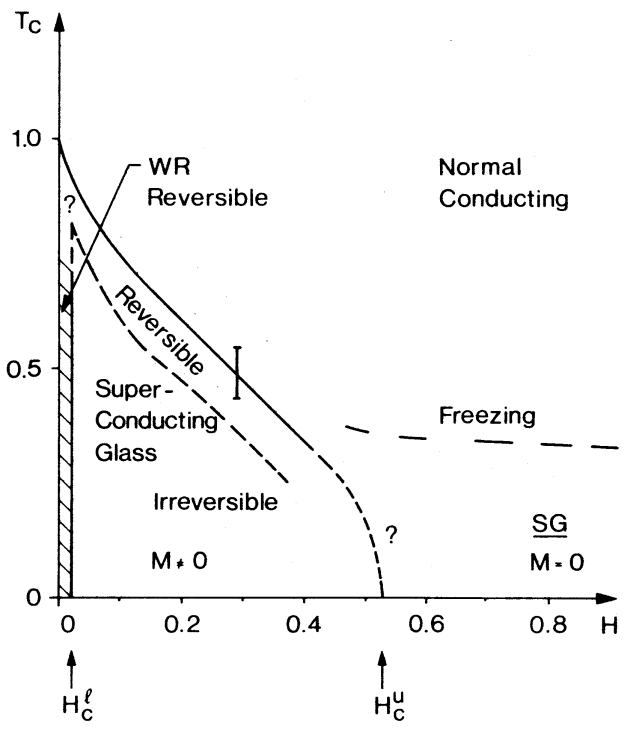

FIG. 2. Phase diagram in $H-T$ plane. Reversible weak-random $\left(\mathrm{WR}\right.$ ) phase between $H \approx 0.0$ and $H_{c}^{l} \approx 0.01$ - 0.03. Between $H_{c}^{l}$ and $H_{c}^{u} \approx 0.5$, superconducting glass phase. Irreversible effects are separated from reversible effects by dashed line. Above $H_{c}^{u}$, usual XY-spin glass phase (SG). Dashed line indicates freezing transition. (analogous to figure 10 in [1])

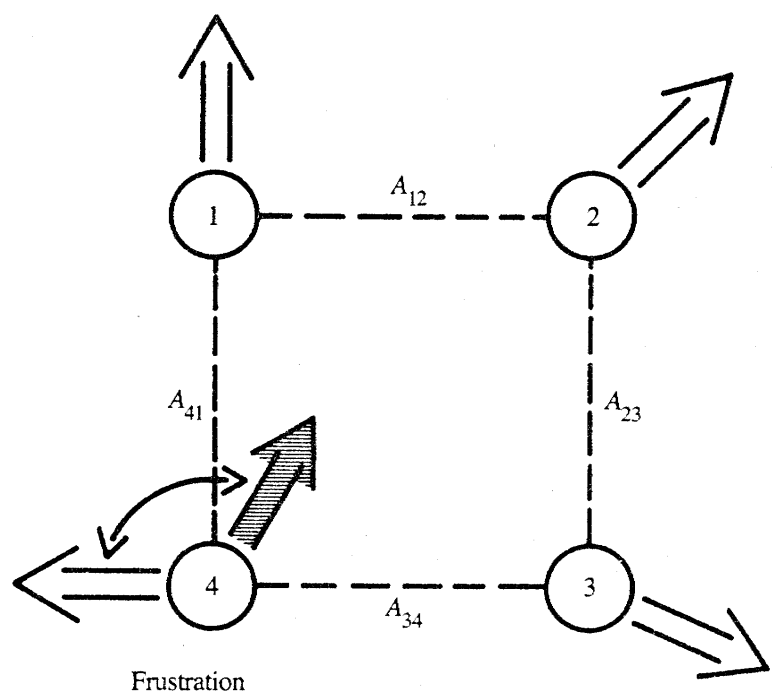

FIG. 3. Plaquette with domains of constant phase $\phi_{i}$ and phase differences $A_{i j}$ (see text)

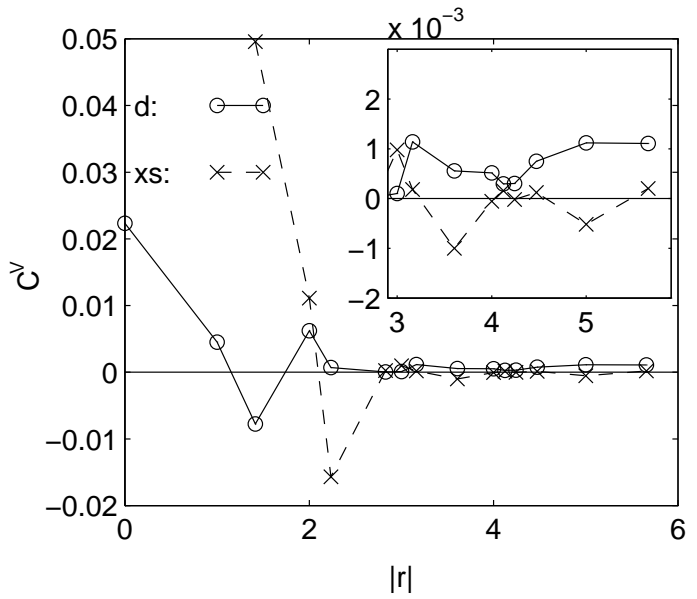

FIG. 4. Vertex-CF versus Cooper pair distance $|r|$ for extended s-wave $(x s)$ and $\mathrm{d}_{x^{2}-y^{2}}$-wave $(d)$ for a $8 \times 8 \times 1$ system with $U=2, t^{\prime}=-0.22$ and $\langle n\rangle=0.78$. (Inset shows the long range regime enlarged.)

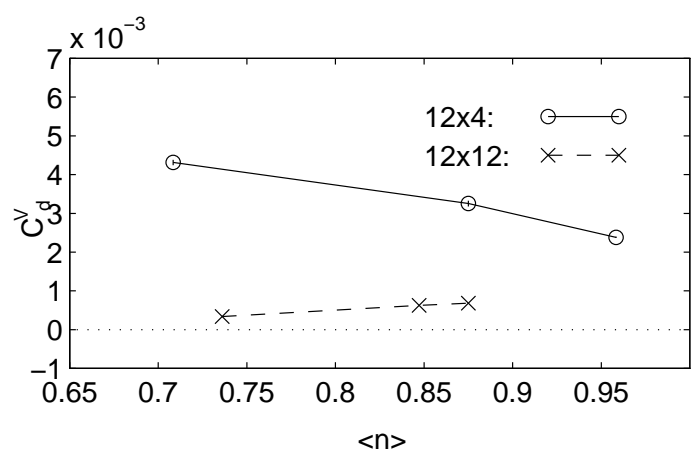

FIG. 5. Averaged $\left(\left|r_{c}\right|=1.9\right)$ vertex CF versus filling for the $\mathrm{tt}^{\prime}-\mathrm{HM}$ with $U=2, t^{\prime}=-0.22$ and the system sizes $12 \times 4$, and $12 \times 12$. The parameter of the PQMC were $\theta=8$ and $\tau=0.125$. Lines are to guide the eye. 


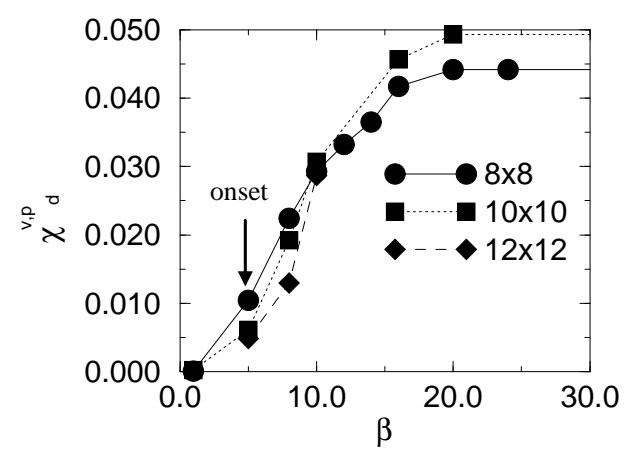

FIG. 6. Cumulated d-wave Vertex-CF $\chi_{d}^{V, P}$ versus inverse temperature $\beta$ for the two-dimensional Hubbard model with $U=2, t^{\prime}=-0.22$ and $\langle n\rangle \approx 0.8$. (data from figure 5.10 in [33].)

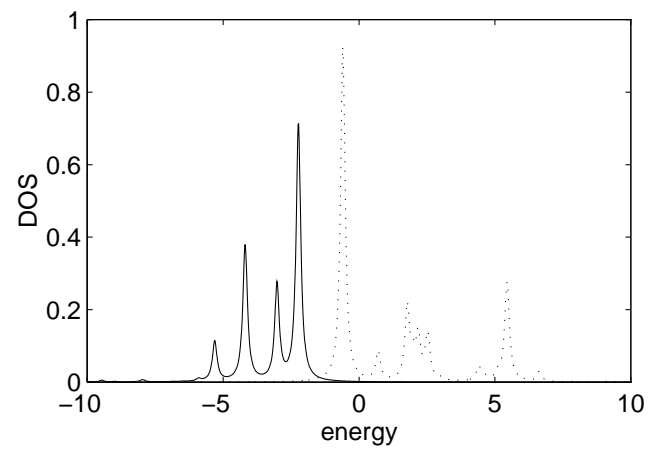

FIG. 7. Density of states (DOS) for the two-dimensional Hubbard model with $U=2, t^{\prime}=-0.22$ and $\langle n\rangle \approx 0.75$ and $L=8$. Energy axis in units of $t$. (data from [46]) 